\title{
Las nebulizaciones con solución salina hipertónica al 5\% disminuyen las exacerbaciones respiratorias en pacientes pediátricos traqueostomizados
}

\author{
CLAUDIA ASTUDILLO M.*, GREGORY VILLARROEL S.**, MARÍA DE LOS ÁNGELES PAUL D.***, \\ VALERIA TRINCADO B.****, PATRICIO BARAÑAO G.***** y MIREYA MÉNDEZ R.*
}

\begin{abstract}
Nebulized 5\% hypertonic saline decreases respiratory exacerbations in pediatric patients with tracheostomy
\end{abstract}

Nebulized hypertonic saline solution is useful in pediatric diseases such as bronchiolitis and cystic fibrosis. However its usefulness in pediatric patients with tracheostomy has not been studied. Our aim was to determine the usefulness of nebulized hypertonic solution 5\% (SSH5\%) in this population. Methods: Prospective cross over, double-blind, randomized, placebo-controlled study. It was approved by ethics committee. 34 out of 37 tracheostomized pediatric patients hospitalized from February to May 2013, were selected for this study. They underwent a period of wash out a month later. They were randomly divided into a treatment group and a placebo group. Treatment group received hypertonic saline solution $5 \%(\mathrm{SSH} \%$ ) and placebo group saline solution $0.9 \%(\mathrm{SSF})$. Both groups received nebulization once daily for 30 days. Then there was a second washout with subsequent crossover. Data collected were: patients identification, number of aspirations per day, type of aspirated secretions, mucus plug, fever, oxygen flow supplied and pressure of mechanical ventilator. The first nebulization on group SSH5\% was defined as a tolerance test. Data analysis was performed using chi-square, Mann-Whitney and $t$ Student tests. The main measured effect was presence and number of respiratory exacerbations, evaluated with Prescott test. Results: $50 \%$ of patients were female, their mean age was 3.94 years-old and $82 \%$ were with mechanical ventilation. We did not find significant differences between both groups in age, sex or mechanical ventilation. Treatment group SSH5\% had less number of respiratory exacerbations than SSF group $(p=0.00595)$. No adverse effects were observed with the use of SSH5\%. Conclusions: Nebulized SSH5\% decreased pulmonary exacerbations in patients with tracheostomy with or without mechanical ventilation, being safe its application.

Key words: Saline solution, hypertonic; child, preschool; tracheostomy; ventilators, mechanical; тисиs.

\section{Resumen}

El uso de nebulizaciones de solución salina hipertónica es beneficioso en enfermedades pediátricas, como bronquiolitis y fibrosis quística, sin embargo, no se ha estudiado su efecto en pacientes pediátricos traqueostomizados. Nuestro propósito fue determinar la utilidad de las nebulizaciones con solución hipertónica al 5\% (SSH5\%) en esta población. Pacientes y Método: Estudio prospectivo 'cross over', doble ciego, aleatorizado, controlado por placebo. Aprobado por comité de ética. De 37 pacientes traqueostomizados internados en el Hospital Josefina Martínez desde febrero a mayo de

* Pediatra Broncopulmonar, Hospital Josefina Martínez. Instructor Adjunto, Departamento de Cardiología y Enfermedades Respiratorias, División de Pediatría. Escuela de Medicina, Pontificia Universidad Católica de Chile.

** Kinesiólogo, Hospital Josefina Martínez. Instructor Adjunto, Escuela de Ciencias de la Salud. Pontificia Universidad Católica de Chile.

*** Pediatra. Hospital Josefina Martínez.

**** Enfermera. Hospital Josefina Martínez.

*****Kinesiólogo, Hospital Josefina Martínez. Profesor Adjunto, Escuela de Ciencias de la Salud. Pontificia Universidad Católica de Chile. 
2013, se seleccionaron 34 que fueron sometidos a un periodo de 'wash out' de un mes, posteriormente se dividieron en forma aleatoria, en grupo tratamiento y grupo placebo. El grupo tratamiento recibió solución salina hipertónica al 5\% (SSH5\%) y el grupo placebo solución salina al 0,9\% (SSF). Ambos grupos recibieron nebulización una vez al día durante 30 días. Luego, hubo un segundo período de 'wash out'con posterior cross over. Los datos recolectados fueron: identificación del paciente, número de aspiraciones/día, tipo de secreciones aspiradas, tapón mucoso, fiebre, flujo de oxígeno suministrado y presiones del ventilador mecánico. La primera nebulización grupo SSH5\% se definió como prueba de tolerancia. El análisis de datos se realizó con pruebas chi cuadrado, Mann-Whitney y t de Student. El efecto principal medido fue presencia y número de exacerbaciones respiratorias, evaluado con test de Prescott. Resultados: 50\% de los pacientes fueron de sexo femenino, su media de edad fue 3,94 años y $82 \%$ de ellos estaban con ventilación mecánica. No encontramos diferencias significativas en edad, sexo o uso de ventilación mecánica entre ambos grupos. El grupo tratamiento SSH5\% presentó menor número de exacerbaciones respiratorias que el grupo SSF $(p=0,00595)$. No se observaron efectos adversos con el uso de SSH5\%. Conclusiones: Las nebulizaciones SSH5\% disminuyeron las exacerbaciones respiratorias en pacientes traqueostomizados con y sin ventilación mecánica, siendo segura su aplicación.

Palabras clave: Solución salina hipertónica, niños, traqueostomía, ventilación mecánica, mucus.

\section{Introducción}

En los niños con enfermedad respiratoria crónica el clearance mucociliar se encuentra afectado por múltiples mecanismos que incluyen: la producción aumentada de moco, la presencia de infecciones del tracto respiratorio, la composición anormal del moco y la pérdida de células ciliadas $^{1,2}$, lo que favorece la inflamación e infección de la vía aérea. Esto, sumado a la presencia de la cánula de traqueostomía ${ }^{3}$, explica el riesgo de infecciones de vía aérea baja en pacientes traqueostomizados aumentando la morbimortalidad.

La solución salina hipertónica nebulizada se ha utilizado para facilitar el manejo de secreciones en pacientes con enfermedad respiratoria, mejorando la reología del moco en los bronquiolos, rompiendo los enlaces iónicos dentro del gel de moco, disminuyendo así la viscosidad y elasticidad del mismo, y estimulando los movimientos ciliares $^{4,5}$. Como resultado se forma un moco más compactado e hidratado que puede ser removido más facilmente por los cilios ${ }^{5,6}$.

Las propiedades de la solución salina hipertónica, además de su bajo costo, han llevado a su uso en la práctica clínica demostrando su utilidad en patologías agudas, como la bronquiolitis y en crónicas como, fibrosis quística y bronquiectasias no secundarias a fibrosis quistica. En lactantes con bronquiolitis leve y moderada se ha observado una disminución en los sintomas y en el tiempo de hospitalización, al compararla con solución salina fisiológica ${ }^{7-9}$. En pacientes con fibrosis quística la solución salina hipertónica se utiliza hace varios años, en concentraciones desde 3 a $7 \%$, sin mayores efectos adversos. Wark et al. demostraron que el tratamiento después de $48 \mathrm{~h}$ por dos veces al día reduce los episodios de infección, mejorando la función pulmonar, la calidad de vida de los pacientes y la asistencia al colegio y al trabajo ${ }^{6}$. En pacientes adultos portadores de bronquiectasias no secundarias a fibrosis quística, se ha observado que el uso regular de solución salina al $7 \%$ es seguro ${ }^{10}$ mejora la función pulmonar, calidad de vida y reduce la utilización del sistema de salud ${ }^{1}$.

El Hospital Josefina Martínez es un centro de referencia para el manejo de pacientes con enfermedades respiratorias crónicas de diferentes etiologías que incluyen enfermedades neuromusculares, del sistema nervioso central y del parénquima pulmonar y/o vía aérea. El $90 \%$ de los pacientes poseen traqueostomía y un $70 \%$ asistencia ventilatoria. No existen reportes en la literatura de la utilidad de nebulizaciones con solución salina hipertónica en pacientes pediátricos traqueostomizados, sin embargo, nuestra hipótesis es que esta población podría beneficiarse con su uso.

El objetivo de este estudio fue determinar la utilidad de las nebulizaciones con solución salina hipertónica al $5 \%$ en el manejo de pacientes pediátricos traqueostomizados con ventilación mecánica crónica o sin ésta.

\section{Pacientes y Método}

Se diseñó un estudio prospectivo cross over, doble ciego, aleatorio, controlado por placebo. Se incluyeron los pacientes pediátricos traqueostomizados hospitalizados en el Hospital Josefina Martínez, durante febrero a mayo de 2013, a 
cuyos padres se les solicitó firmar un consentimiento informado para participar en el estudio.

A todos los pacientes se les realizó una prueba de tolerancia previa al inicio del estudio, en la que se administró una nebulización $\mathrm{SSH} 5 \%$ durante 10 min y luego se constató, por un único evaluador entrenado, la aparición de sibilancias luego de esta prueba.

De un total de 37 pacientes, en dos de ellos sus padres no firmaron el consentimiento informado $\mathrm{y}$ uno fue excluido por presentar limitación del esfuerzo terapéutico (establecido por el comité de ética de su hospital de origen). Los 34 pacientes restantes aprobaron la prueba de tolerancia.

El estudio fue aprobado por el comité de ética del Servicio de Salud Metropolitano Suroriente y financiado por la Fundación Josefina Martínez.

\section{Método}

Un total de 34 pacientes fueron sometidos a un período de limpieza o wash-out antes de iniciar la aplicación de las nebulizaciones determinadas para el estudio, con el fin de eliminar posibles efectos residuales que existieran en aquellos pacientes que hayan sido intervenidos con nebulizaciones hipertónicas de forma rutinaria.

Luego del wash-out, los participantes fueron divididos de forma aleatoria, por una enfermera del hospital que no es parte del grupo investigador, en dos grupos: Grupo tratamiento que recibió $\mathrm{SSH}$ al $5 \%$ y grupo placebo que recibió una nebulización con solución salina al $0,9 \%$ (SSF). Se cegó al personal que administraba y al paciente que recibía la nebulización.

Ambos grupos recibieron la nebulización una vez al día durante 30 días (Figura 1).

Las nebulizaciones se realizaron a través de un sistema Jet de pequeño volumen con un nebulizador Hudson ${ }^{\circledR}$ con $4 \mathrm{ml}$ de la solución correspondiente para cada grupo, por un período de tiempo no mayor a $10 \mathrm{~min}$, con un flujo de oxígeno de 8 litros por minuto. El paciente se posicionó en sedente o en posición de Fowler con el nebulizador ubicado en forma vertical (Figura 2).

$\mathrm{Al}$ cabo de los primeros 30 días de intervención se realizó un segundo wash-out por un mes para eliminar los posibles efectos obtenidos en ambos grupos. Luego de este segundo wash-out se realizó el cross over en donde el grupo que durante la primera intervención recibió SSH5\% ahora empezó a recibir nebulización SSF, y viceversa.

Variables de evaluación: número de exacerbaciones respiratorias, calidad y cantidad de las secreciones traqueales, número de aspiraciones por día, presencia de tapón mucoso y oclusión parcial o total de la cánula.

Exacerbaciones respiratorias: se contabilizó el número de exacerbaciones en cada paciente para cada período de nebulización al que fue sometido. Se definió exacerbación respiratoria de acuerdo a los elementos indicados en la Tabla 1. Esta definición incluyó traqueítis, bronquitis y neumonía.

Secreciones endotraqueales: fueron valoradas a través del registro realizado por el personal de enfermería y/o kinesiología del hospital.

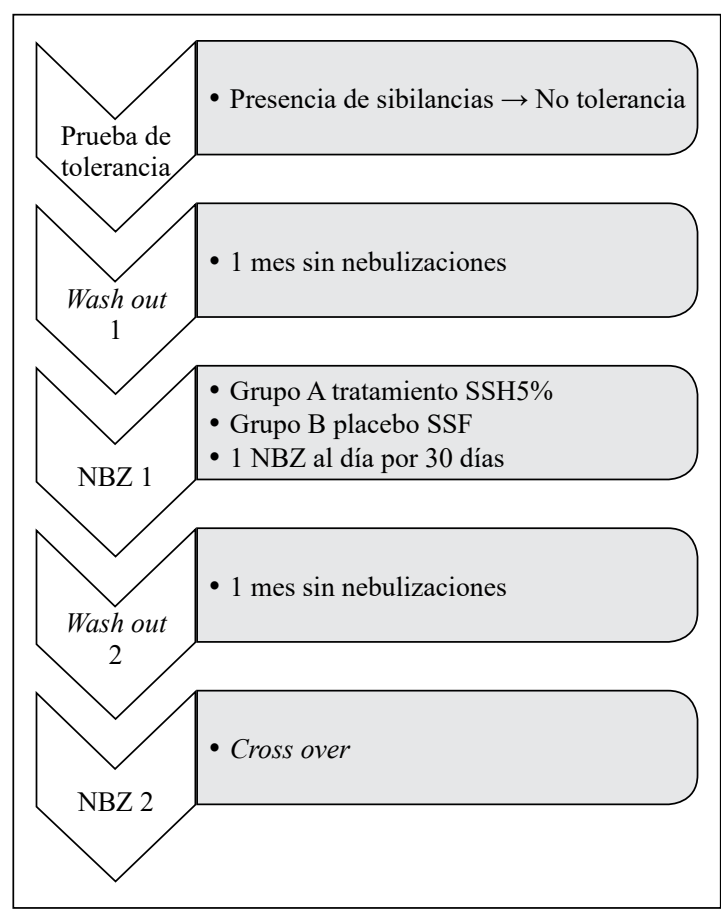

Figura 1. Flujograma de intervención del estudio. NBZ: nebulización.

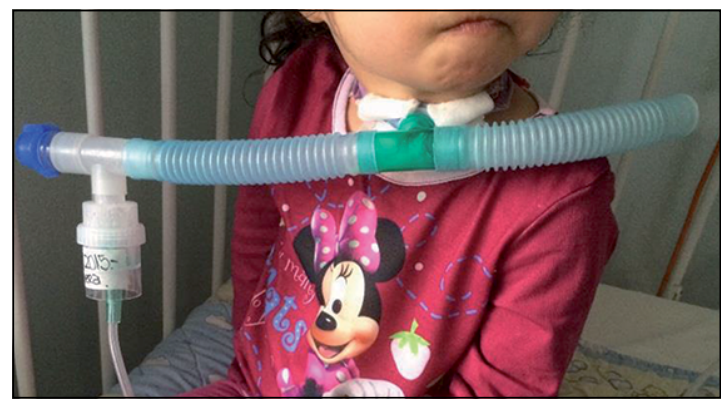

Figura 2. Nebulizador en T conectado a traqueostomía, que fue empleado para las nebulizaciones. 


\section{Tabla 1. Criterios de exacerbación respiratoria}

Presencia de fiebre (temperatura rectal $>38^{\circ} \mathrm{C}$ ) más presencia de signos y síntomas de patología respiratoria, con uno o más de los siguientes condicionantes:

1. Aumento de los requerimientos de oxígeno adicional Medido en litros por minuto

2. Aumento de las presiones basales del ventilador mecánico $\quad$ Medida en $\mathrm{cm} \mathrm{de} \mathrm{H}_{2} \mathrm{O}$

3. Cambio en las imágenes radiológicas previas Radiografía de tórax

La calidad de las secreciones fue categorizada en relación a la coloración de las secreciones como: mucosas, purulentas, hemáticas y las combinaciones entre éstas.

La cantidad de secreciones fue categorizada como se describe en la Tabla 2.

Se registró la presencia de tapones mucosos al aspirar a través de la cánula de traqueostomía, así como la oclusión de la misma.

\section{Análisis estadístico}

Todos los datos fueron analizados a través del software SPSS 17.0. El análisis de las variables paramétricas se realizó a través de la prueba de t-student y $\chi^{2}$. Las variables no paramétricas fueron analizadas por medio de la prueba $U$ de Mann-Whitney. Fijamos niveles de $p<0,05$ para establecer significancia.

Para evaluar el efecto carry-over en los períodos de tiempo, se utilizó la prueba de Mainland-Gart y para la diferencia de tratamiento el Prescott test, considerando un valor significativo de $\mathrm{p}<0,05$ en la Prueba de Fisher.

\section{Resultados}

Se estudiaron treinta y cuatro pacientes $(n=34)$, cuyas características demográficas se
Tabla 2. Cantidad de secreciones

\begin{tabular}{|cc|}
\hline+ & Poca \\
++ & Moderada \\
+++ & Alta \\
\hline
\end{tabular}

describen en la Tabla 3. No se encontró diferencias significativas en la distribución del sexo y la edad de los grupos estudiados, ni tampoco en relación al uso o no uso de ventilación mecánica.

Las variables número de aspiraciones y presencia de tapón mucoso en las secreciones aspiradas, no mostraron diferencias significativas con el uso de SSH5\% versus SSF (Tabla 4).

Los resultados obtenidos en relación a los eventos de oclusión de cánula y al número de exacerbaciones durante el estudio, demuestran que hubo una reducción significativa del número de las exacerbaciones respiratorias en aquellos pacientes que recibieron nebulización SSH5\%, independiente del período estudiado (Tabla 5) y que no hubo cambios significativos en relación a la oclusión de cánula con el uso de una solución u otra (Tabla 6).

Debemos destacar que en este estudio clínico no se registraron efectos adversos.

Tabla 3. Datos demográficos de los 34 niños traqueostomizados incluidos en el estudio

\begin{tabular}{|lc}
\hline Sexo (\% Hombres) & $17(50 \%)$ \\
Edad (meses) & $47,38 \pm 26,73$ \\
Diagnósticos & $\mathbf{( n = 3 4 )}$ \\
$\quad$ Alteraciones del parénquima pulmonar y vía aérea & 11 \\
Obstrucción vía aérea superior & 14 \\
Enfermedades neuromusculares & 7 \\
Relacionadas con alteraciones del Sistema Nervioso Central & 2 \\
Uso de ventilación mecánica & $\mathbf{n}$ \\
Traqueostomía + ventilación mecánica & 28 \\
Traqueostomía & 6 \\
Total & 34 \\
\hline
\end{tabular}


Tabla 4. Efecto de nebulizaciones SSF y SSH5\% sobre número de aspiraciones y tapones mucosos

\begin{tabular}{|lccc|}
\hline Variable & SSH5\% & SSF 0,9\% & Valor de p \\
\hline n de aspiraciones & $71,94 \pm 20,24$ & $70,57 \pm 19,23$ & 0,455 \\
n de tapones mucosos & $5,47 \pm 7,90$ & $4,09 \pm 4,47$ & 0,1919 \\
\hline
\end{tabular}

SSF: Solución salina al 0,9\%. SSH 5\%: Solución salina hipertónica al 5\%. Cada valor representa la media \pm su DS.

Tabla 5. Efecto de nebulizaciones SSF y SSH5\% sobre el número de exacerbaciones

\begin{tabular}{|lccc|}
\hline Grupo & $\begin{array}{c}\text { n de exacerbaciones } \\
\text { en período 1 }\end{array}$ & $\begin{array}{c}\text { Sin exacerbaciones } \\
\text { en ambos períodos }\end{array}$ & $\begin{array}{c}\text { n de exacerbaciones } \\
\text { en período 2 }\end{array}$ \\
\hline SSF & 5 & 10 & 1 \\
SSH5\% & 0 & 18 & 0 \\
\hline
\end{tabular}

$\mathrm{p}=0,000595 *$ Prueba de Fisher (Prescott's Test): SSF: Solución Salina al 0,9\%. SSH 5\%: Solución Salina Hipertónica al $5 \%$.

Tabla 6. Efecto de nebulizaciones SSF y SSH5\% sobre el número de oclusiones de cánulas

\begin{tabular}{|lccc|}
\hline Grupo & $\begin{array}{c}\text { n oclusiones de cánula } \\
\text { en período 1 }\end{array}$ & $\begin{array}{c}\text { Sin oclusión de cánula } \\
\text { en ambos períodos }\end{array}$ & $\begin{array}{c}\text { n oclusión de cánula } \\
\text { en período } 2\end{array}$ \\
\hline SSF 0,9\% & 5 & 10 & 1 \\
\hline SSH5\% & 0 & 18 & 0 \\
\hline
\end{tabular}

$\mathrm{p}=0,4884$ Prueba de Fisher (Prescott's Test): SSF: Solución Salina al 0,9\%. SSNH 5\%: Solución Salina Hipertónica al $5 \%$.

\section{Discusión}

En nuestro conocimiento este ensayo clínico randomizado, controlado sería el primero en examinar el efecto de las nebulizaciones con solución salina hipertónica al 5\% en niños traqueostomizados. Este tratamiento fue bien tolerado y sin efectos adversos para este grupo de pacientes, al igual que lo anteriormente demostrado para bronquiolitis viral ${ }^{11}$.

En nuestro estudio no observamos disminución en el número de aspiraciones, en la presencia de tapón mucoso o en eventos de oclusión de cánula en el grupo tratado. Sabemos que la cantidad y calidad de las secreciones es una variable subjetiva, de allí que el número de aspiraciones corresponde a la apreciación de su necesidad por parte del personal de salud que no necesariamente tiene relación con la característica del moco. En relación al tapón mucoso y la oclusión de cánula nuestros hallazgos podrían ser explicados por un sub-registro, esto secundario al grado de experiencia de nuestro personal, quien resuelve esta situación rápidamente y en forma rutinaria.

La disminución observada en el número de exacerbaciones que alcanzó significancia estadística podría atribuirse a la estandarización de la técnica utilizada para la nebulización, llevada a cabo por personal debidamente adiestrado sumado a los efectos propios de la solución hipertónica descritos en la literatura que podrían contribuir con una mejoría en el clearance mucociliar de la vía aérea ${ }^{8}$.

El hospital Josefina Martínez es un centro asistencial que concentra una gran cantidad de pacientes traqueostomizados quienes permanecen hospitalizados por tiempo prolongado permitiendo un mejor control de las intervenciones en el período estudiado.

Una limitación descrita en otros estudios, es la pérdida del ciego debida a la percepción del sabor salado de la nebulización por parte del paciente. Es nuestro estudio no existe esta limitación dado que la administración se realizó exclusivamente a través de la traqueostomía ${ }^{12}$.

No evaluamos función pulmonar en este grupo, debido a su corta edad y a la dificultad técnica al portar una cánula de traqueostomía. En un estudio realizado en lactantes y preescolares con fibrosis quística tratados con SSH al $6 \%$ versus SSF se utilizó el índice de aclaramiento pulmo- 
nar (LCI) como una forma de evaluar función pulmonar ${ }^{13}$. Eventualmente esta técnica podría adaptarse para futuros estudios en pacientes traqueostomizados.

En conclusión, las nebulizaciones con solución salina hipertónica al 5\% disminuyeron las exacerbaciones respiratorias en pacientes traqueostomizados con y sin ventilación mecánica, siendo segura su aplicación.

\section{Bibliografía}

1.- KELLETT F, ROBERT N M. Nebulised 7\% hypertonic saline improves lung function and quality of life in bronchiectasis. Respir Med 2011; 105: 1831-5.

2.- BOOGAARD R, DE JONGSTE J C, MERKUS P J F M. Pharmacotherapy of impaired mucociliary clearance in non-CF pediatric lung disease. A review of the literature. Pediatr Pulmonol 2007; 42: 989-1001.

3.- GARCÍA R. BELTRÁN C, CHATEAU B. Infecciones respiratorias asociadas a traqueostomias en niños. Neumol Pediátrica 2011; 6: 134-7.

4.- BALAGUER SANTAMARÍA A BAJ. El suero hipertónico Nebulizado puede disminuir el ingreso hospitalario en lactantes con bronquiolitis aguda. Evidencias en Pediatría 2009; 5: 2005-7.

5.- ELKINS M R, BYE P T P. Mechanisms and applications of hypertonic saline. J R Soc Med 2011; 104 Suppl: S25.

6.- WARK P, MCDONALD V M. Nebulised hypertonic saline for cystic fibrosis. Cochrane Database Syst Rev
2009; 2015 (5). doi: 10.1002/14651858.CD001506. pub3.

7.- KUZIK B A, AL QADHI S A, KENT S, FLAVIN M P, HOPMAN W, HOTTE S, et al. Nebulized hypertonic saline in the treatment of viral bronchiolitis in infants. $\mathrm{J}$ Pediatr 2007; 151 (C).

8.- ZHANG L, MENDOZA-SASSI R A, WAINWRIGHT C, KLASSEN T P. Nebulized hypertonic saline solution for acute bronchiolitis in infants. Cochrane Database Syst Rev 2008; (July 2015). doi: 10.1002/14651858. CD006458.pub2.

9.- LUO Z, FU Z, LIU E, XU X, FU X, PENG D, et al. Nebulized hypertonic saline treatment in hospitalized children with moderate to severe viral bronchiolitis. Clin Microbiol Infect 2011; 17 (i): 1829-33.

10.- KELLETT F, REDFERN J, MCNIVEN R. Evaluation of nebulised hypertonic saline $(7 \%)$ as an adjunct to physiotherapy in patients with stable bronchiectasis. Respir Med 2005; 99: 27-31.

11.- AL-ANSARI K, SAKRAN M, DAVIDSON B, EL SAYYED R, MAHJOUD H, IBRAHIM K. Nebulized $5 \%$ or $3 \%$ Hypertonic or $0.9 \%$ saline for treating acute bronchiolitis in infants. J Pediatr 2010; 157: 630-4.

12.- NICOLSON C, STIRLING B, BORG B, BUTTON B, WILSON J, HOLLAND A. The long term effect of inhaled hypertonic saline $6 \%$ in non-cystic fibrosis bronchiectasis. Resp Med 2012; 106: 661-6.

13.- SUBBARAO P, STANOJEVIC S, BROWN M, JENSEN R, ROSENFELD M, DAVIS S, et al. Lung clearance index as an outcome measure for clinical trials in young children with cystic fibrosis. Am J Respir Crit Care Med 2013; 188: 456-60.
Correspondencia a:

Dra. Claudia Astudillo M.

Hospital Josefina Martínez, Departamento de Cardiología y Enfermedades Respiratorias

División de Pediatría, Escuela de Medicina. Pontificia

Universidad Católica de Chile.

E-mail: claudiastu@gmail.com 